\title{
Dynamic Expression of Tenascin-C After Myocardial Ischemia and Reperfusion: Assessment by ${ }^{125}$ I-Anti-Tenascin-C Antibody Imaging
}

Junichi Taki ${ }^{1}$, Anri Inaki ${ }^{1}$, Hiroshi Wakabayashi ${ }^{1}$, Kyoko Imanaka-Yoshida ${ }^{2}$, Kazuma Ogawa $^{3}$, Michiaki Hiroe ${ }^{4}$, Kazuhiro Shiba ${ }^{5}$, Toshimichi Yoshida ${ }^{2}$, and Seigo Kinuya ${ }^{1,6}$

${ }^{1}$ Department of Nuclear Medicine, Kanazawa University Hospital, Kanazawa, Japan; ${ }^{2}$ Department of Pathology, Mie University School of Medicine, Mie, Japan; ${ }^{3}$ Graduate School of Natural Science and Technology, Kanazawa University, Kanazawa, Japan; ${ }^{4}$ Department of Nephrology and Cardiology, International Medical Center of Japan, Tokyo, Japan; ${ }^{5}$ Division of Tracer Kinetics, Advanced Science Research Center, Kanazawa University, Kanazawa, Japan; and ${ }^{6}$ Department of Biotracer Medicine, Kanazawa University Graduate School of Medical Sciences, Kanazawa, Japan

Tenascin-C, an extracellular matrix glycoprotein, appears only in the early stages of embryonic development. It is not normally expressed in the adult heart but does reappear transiently in distinct areas in association with active tissue remodeling. The aim of this study was to explore serial changes in the expression of tenascin- $\mathrm{C}$ after myocardial ischemia and reperfusion, using 125/-labeled anti-tenascin-C antibody (125J-TNC-Ab) in a rat model of acute ischemia and reperfusion. Methods: The left coronary artery was occluded for 20 or $30 \mathrm{~min}$, followed by reperfusion for 1, 3, or $7 \mathrm{~d}$ in rats with $20 \mathrm{~min}$ of ischemia and for 1, 3, 7, 14 , or $28 \mathrm{~d}$ in rats with $30 \mathrm{~min}$ of ischemia. At the time of the study, 125I-TNC-Ab (1.0-2.5 MBq) was injected. Three to $5 \mathrm{~h}$ later, to verify the area at risk, ${ }^{99 m T c-m e t h o x y i s o b u t y l i s o n i t r i l e ~(100-200 ~}$ $\mathrm{MBq}$ ) was injected intravenously just after the left coronary artery reocclusion and the rats were sacrificed $1 \mathrm{~min}$ later. Dual-tracer autoradiography was performed to assess ${ }^{125}$ I-TNC-Ab uptake and the area at risk. Results: In rats with $20 \mathrm{~min}$ of ischemia, 125I-TNC-Ab uptake peaked at $3 \mathrm{~d}$ after reperfusion, followed by faint uptake after $7 \mathrm{~d}$ (uptake ratios at 1, 3, and $7 \mathrm{~d}$ after reperfusion were $1.81 \pm 0.53,2.46 \pm 0.79$, and $1.23 \pm 0.17$, respectively $[P<0.05$ vs. $3 \mathrm{~d}]$ ). In rats with $30 \mathrm{~min}$ of ischemia, uptake was high at 1 and $3 \mathrm{~d}$ after reperfusion $(2.99 \pm 0.90$ and $2.71 \pm$ 0.80 , respectively), decreased at 7 and $14 \mathrm{~d}(1.94 \pm 0.23$ and $2.06 \pm 0.37$, respectively), and was weak at $28 \mathrm{~d}(1.47 \pm 0.27$, $P<0.005$ vs. $1 \mathrm{~d}, P<0.05$ vs. $3 \mathrm{~d})$. Conclusion: These data indicate that ${ }^{125}$ I-TNC-Ab imaging may be a way to monitor myocardial injury and its repair process after ischemia and reperfusion by visualizing tenascin- $\mathrm{C}$ expression.

Key Words: tenascin-C; ${ }^{125}$ I-anti-tenascin-C-antibody; myocardial ischemia; reperfusion; autoradiography

J Nucl Med 2010; 51:1116-1122

DOI: 10.2967/jnumed.109.071340

Received Oct. 5, 2009; revision accepted Mar. 17, 2010.

For correspondence or reprints contact: Junichi Taki, Department of Nuclear Medicine, Kanazawa University Hospital, 13-1 Takara-machi, Kanazawa, 920-8641, Japan.

E-mail: taki@med.kanazawa-u.ac.jp

COPYRIGHT $\odot 2010$ by the Society of Nuclear Medicine, Inc.
C ardiac repair after myocardial infarction is regulated thorough activation and repression of the acute inflammatory process, followed by timely infiltration of the infarcted myocardium with myofibroblasts. These secrete large amounts of extracellular matrix proteins, leading to formation of collagen-based scar tissue in the infarct. Inflammatory and fibrotic processes are critically involved in the pathogenesis of ventricular remodeling, which is a significant predictor of left ventricular dysfunction and an adverse prognosis (1). Extracellular matrix and cardiac cells are essential for maintaining the integrity of cardiac tissue and play an important role not only in structural and mechanical support but also in modulation of cell function.

It has been reported that tenascin-C, an extracellular matrix glycoprotein, could provide important biologic signaling that influences cell motility, proliferation, differentiation, survival, or apoptosis via cellular-extracellular matrix interaction during remodeling of various tissues $(2,3)$. Tenascin- $C$ appears in the heart only in the early stages of embryonic development and is not normally expressed in adults. However, it reappears transiently in association with myocardial injury such as myocardial infarction (4-6), hibernating myocardium (7), myocarditis $(6,8,9)$, and dilated cardiomyopathy $(10,11)$. Its site-specific expression suggests that tenascin- $C$ plays important roles during tissue remodeling but also that it can serve as an indicator of myocardial disease activity.

Recently, we and other groups have reported increased serum tenascin- $\mathrm{C}$ in heart failure patients, reflecting the severity and progression of ventricular remodeling (12-15). Of particular interest is that patients with acute myocardial infarction with high serum levels of tenascin-C within $1 \mathrm{wk}$ of infarction have a greater incidence of ventricular remodeling 6 mo later and a worse long-term prognosis 
(16), indicating that tenascin-C can be a possible predictor of ventricular remodeling and poor prognosis after myocardial infarction. These considerations make it important to assess the expression of tenascin- $\mathrm{C}$ in the acute phase of myocardial infarction.

In previous animal studies on acute myocardial infarction induced by permanent ligation of a coronary artery, it was reported that tenascin-C appears transiently during the acute stage, plays several significant roles in myocardial tissue remodeling, and may be a potential candidate for molecular imaging $(9,17)$. For clinical application of the imaging, it is essential to analyze the molecular dynamics in the model of myocardial ischemia and reperfusion that is the general scenario in the era of percutaneous coronary intervention in acute coronary syndrome.

The aim of this study was to explore spatial and temporal changes in the expression of tenascin- $\mathrm{C}$ after myocardial ischemia and reperfusion using ${ }^{125} \mathrm{I}$-labeled anti-tenascin-C antibody ( $\left.{ }^{125} \mathrm{I}-\mathrm{TNC}-\mathrm{Ab}\right)$ in a rat model of acute ischemia and reperfusion.

\section{MATERIALS AND METHODS}

\section{Animal Model of Acute Ischemia and Reperfusion}

All experimental procedures involving animals were conducted in accordance with the institutional guidelines set by the Institute for Experimental Animals, Kanazawa University Advanced Science Research Center. Ten- to 14-wk-old male Wistar rats $(n=$ 51) were anesthetized with an intraperitoneal administration of 40 mg of pentobarbital per kilogram of body weight and ventilated mechanically with room air. After left thoracotomy and exposure of the heart, a 7-0 polypropylene suture on a small curved needle was passed through the myocardium beneath the proximal portion of the left coronary artery (LCA), and both ends of the suture were passed through a small vinyl tube to make a snare. The suture material was pulled tightly against the vinyl tube to occlude the LCA. Myocardial ischemia was confirmed by regional cyanosis of the myocardial surface and ST-segment elevation on the electrocardiograph. Two series of experiments were performed. The first evaluated a 20-min interval of LCA occlusion and reperfusion, and the second evaluated a 30-min interval of LCA occlusion and reperfusion, to determine the distribution of tenascin- $\mathrm{C}$ expression. Reperfusion was obtained by release of the snare and was confirmed by a myocardial blush over the area at risk. The snare was left loose on the surface of the heart until repeated thoracotomy for reocclusion of the LCA just before sacrifice to identify the area at risk (18). Groups of animals with $20 \mathrm{~min}$ of occlusion were studied at $1 \mathrm{~d}(n=6), 3 \mathrm{~d}(n=5)$, and $7 \mathrm{~d}(n=5)$ after reperfusion, and groups of animals with $30 \mathrm{~min}$ of occlusion were studied at $1 \mathrm{~d}(n=6), 3 \mathrm{~d}(n=6), 7 \mathrm{~d}(n=5), 14 \mathrm{~d}(n=6)$, and $28 \mathrm{~d}(n=6)$ after reperfusion. At the time of the study, ${ }^{125} \mathrm{I}$ TNC-Ab (1.0-2.5 MBq) was administered via a tail vein. In 6 rats with $30 \mathrm{~min}$ of ischemia and $3 \mathrm{~d}$ of reperfusion, $1.5-2.0 \mathrm{MBq}$ of ${ }^{125}$ I-labeled nonspecific antibody were injected. Three to $5 \mathrm{~h}$ afterward, 100-200 MBq of ${ }^{99 \mathrm{~m}} \mathrm{Tc}-\mathrm{methoxyisobutylisonitrile}$ (MIBI) were injected via a tail vein just after reocclusion of the proximal portion of the LCA for delineation of the area at risk. One minute later, the rat was euthanized and the heart was removed for analysis. The excised heart was rinsed in saline, frozen in isopentane, cooled in dry ice, and embedded in methyl cellulose. Serial short-axis heart sections $20 \mu \mathrm{m}$ thick were obtained on a cryostat to create a series of rings for autoradiography.

\section{Biodistribution Study}

Biodistribution was studied in rats with $30 \mathrm{~min}$ of ischemia and $3 \mathrm{~d}$ of reperfusion with injection of $1.5-2.0 \mathrm{MBq}$ of ${ }^{125} \mathrm{I}-\mathrm{TNC}-\mathrm{Ab}$. Groups of rats were euthanized at $1 \mathrm{~h}(n=4), 5 \mathrm{~h}(n=5)$, and $24 \mathrm{~h}(n=5)$ after tracer injection. Hearts (nonischemic area and infarcted area), other organs (lung, liver, spleen, kidney, and muscle), and blood were weighed and then counted for radioactivity to calculate the percentage injected dose per gram of tissue.

\section{Radiolabeling of Anti-Tenascin C Antibody}

Anti-tenascin-C mouse IgG Fab', a mouse monoclonal antibody against tenascin-C, clone 4F10TT, was raised by immunization of a tenascin-C-null mouse with purified human tenascin-C as described previously (6).

Anti-tenascin-C antibody was radiolabeled with ${ }^{125}$ I by the chloramine-T method (19). Briefly, ${ }^{125}$ I-sodium iodide solution (37 MBq/10 $\mu \mathrm{L}$; Perkin Elmer) was added to $60 \mu \mathrm{L}$ of antibody in phosphate-buffered saline $(0.32 \mathrm{mg} / \mathrm{mL})$. After mixing, $4 \mu \mathrm{L}$ of chloramine-T aqueous solution $(1 \mathrm{mg} / \mathrm{mL})$ were added. After 15 min at room temperature, the reaction was quenched with $20 \mu \mathrm{L}$ of $\mathrm{Na}_{2} \mathrm{H}_{2} \mathrm{SO}_{5}$. The crude product was purified with a PD-10 column (GE Healthcare U.K., Ltd.) with saline as the eluate. The radiochemical purity of ${ }^{125} \mathrm{I}-\mathrm{TNC}-\mathrm{Ab}$ was determined by thinlayer chromatography, which was performed with silica plates (catalog no. 5553; Merck) and with saline as the developing solvent. Radiochemical purity was defined as the percentage of protein-bound activity, which was assessed by dividing counts at the thin-layer chromatography origin by total thin-layer chromatography counts.

As a nonspecific control antibody, mouse $\mathrm{F}\left(\mathrm{ab}^{\prime}\right)_{2}$ IgG1 (Beckman Coulter, Inc.) was reduced to $\mathrm{Fab}^{\prime}$ with $50 \mathrm{mM}$ 2-mercaptoethylamine in phosphate-buffered saline containing $10 \mathrm{mM}$ ethylenediaminetetraacetic acid at $37^{\circ} \mathrm{C}$ for $1 \mathrm{~h}$. Radiolabeling of the control antibody ( $\mathrm{Fab}^{\prime}$ ) with ${ }^{125} \mathrm{I}$ was performed as described above.

\section{Dual-Tracer Autoradiography}

Dual-tracer autoradiography of the left ventricular short-axis slices was performed for the assessment of ${ }^{125}$ I-TNC-Ab uptake and ischemic area $\left({ }^{99 \mathrm{~m}} \mathrm{Tc}-\mathrm{MIBI}\right.$ image). The first autoradiographic exposure on an imaging plate (BAS-MS; Fuji Film) was performed for 15-20 min to visualize the area at risk as expressed by ${ }^{99 m} \mathrm{Tc}-\mathrm{MIBI}$ distribution at $1-2 \mathrm{~h}$ after sacrifice. Three days later (12 half-lives of ${ }^{99 \mathrm{~m}} \mathrm{Tc}$ ), the second exposure was made for $7 \mathrm{~d}$ to image the distribution of ${ }^{125} \mathrm{I}-\mathrm{TNC}-\mathrm{Ab}$.

\section{Data Analysis}

${ }^{125} \mathrm{I}-\mathrm{TNC}-\mathrm{Ab}$ accumulation was evaluated in 3 myocardial slices at the mid-ventricular level spaced $1 \mathrm{~mm}$ apart from one another. The distribution of the tracers was determined by analysis of the digitized autoradiographs. The photostimulated luminescence in each pixel $(100 \times 100 \mu \mathrm{m})$ was determined using a bioimaging analyzer (BAS-5000; Fuji Film). For quantitative analysis, the uptake values of each region of interest (ROI) were expressed as the background-corrected photostimulated luminescence per unit area $\left(1 \mathrm{~mm}^{2}\right)$. A background ROI was set adjacent to the left ventricle. Ischemic and normally perfused areas were 
defined from the ${ }^{99 \mathrm{~m}} \mathrm{Tc}-\mathrm{MIBI}$ image, and these ROIs were applied to the ${ }^{125} \mathrm{I}-\mathrm{TNC}-\mathrm{Ab}$ images to evaluate the uptake of ${ }^{125} \mathrm{I}-\mathrm{TNC}$ Ab. An area of significant ${ }^{125}$ I-TNC-Ab uptake was also defined manually as an ROI. The ${ }^{125}$ I-TNC-Ab uptake ratio was calculated by dividing the uptake value in an ischemic area by that of a normally perfused area. The ratio of a ${ }^{125} \mathrm{I}-\mathrm{TNC}-\mathrm{Ab}$ uptake ROI to an ischemic ROI was defined as the percentage of the ${ }^{125} \mathrm{I}-\mathrm{TNC}$ Ab uptake area (18). All parameters in each rat were expressed as the average value obtained from the analysis of 3 representative slices.

\section{Immunohistochemical Staining}

Short-axis frozen sections adjacent to the slices for autoradiography were mounted on slides. These short-axis heart sections were washed with phosphate-buffered saline and stained with mouse anti-tenascin-C antibody 4F10TT, the same antibody used for autoradiography, using direct immunoperoxidase technique. For paraffin sections, the heart was fixed in $4 \%$ paraformaldehyde, embedded in paraffin, and immunostained as previously described (5). Briefly, after treatment with pepsin for $10 \mathrm{~min}$ for antigen retrieval, sections were incubated with anti-tenascin- $\mathrm{C}$ antibody 4F10TT and subsequently with peroxidase-conjugated antimouse IgG Fab' (Medical and Biological Lab Co., Ltd.). After washing, diaminobenzidine $/ \mathrm{H}_{2} \mathrm{O}_{2}$ solution was used to demonstrate antibody binding. The site and distribution of the expression of tenascin- $\mathrm{C}$ were examined with the aid of the hematoxylin- and eosin-stained slice adjacent to the immunohistochemically stained slice.

\section{Statistical Analysis}

All results were expressed as mean \pm SD. Statistical analyses were performed using a Macintosh computer (Apple) with JMP software (version 5.0.1J; SAS Institute, Inc.). Groups were compared using the Tukey-Kramer method. A value of $P$ less than 0.05 was considered statistically significant.

\section{RESULTS}

\section{Radiolabeling}

${ }^{125} \mathrm{I}-\mathrm{TNC}-\mathrm{Ab}$ was prepared with high radiochemical yield $(89 \%)$. After purification by PD-10, ${ }^{125} \mathrm{I}-\mathrm{TNC}-\mathrm{Ab}$ showed a radiochemical purity of over $99 \%$.

\section{Area with ${ }^{125}$ I-TNC-Ab Uptake Versus Area at Risk}

The percentages of ${ }^{125} \mathrm{I}-\mathrm{TNC}-\mathrm{Ab}$ uptake areas against areas at risk in rats with $20 \mathrm{~min}$ of ischemia at 1,3 , and $7 \mathrm{~d}$ after reperfusion were similar $(59.5 \% \pm 10.4 \%, 52.9 \% \pm$ $4.0 \%$, and $62.3 \% \pm 3.1 \%$, respectively; not statistically significant) (Fig. 1).

In rats after $30 \mathrm{~min}$ of reperfusion, the percentages of ${ }^{125} \mathrm{I}-\mathrm{TNC}-\mathrm{Ab}$ uptake areas at 1, 3, 7, 14, and $28 \mathrm{~d}$ after reperfusion tended to decrease with longer reperfusion periods $(70.2 \% \pm 8.2 \%, 63.7 \% \pm 4.3 \%, 63.5 \% \pm 3.7 \%$, $61.0 \% \pm 14.3 \%$, and $52.3 \% \pm 13.0 \%$, respectively), but statistical significance was observed only when $1 \mathrm{~d}$ was compared with $28 \mathrm{~d}(P<0.05)$ (Fig. 1).

When ${ }^{125} \mathrm{I}-\mathrm{TNC}-\mathrm{Ab}$ uptake areas against areas at risk were compared between rats with 20 and $30 \mathrm{~min}$ of ischemia, the uptake areas of rats with $30 \mathrm{~min}$ of ischemia were larger than those of rats with $20 \mathrm{~min}$ of ischemia only at $3 \mathrm{~d}$ after reperfusion $(P<0.05)$.

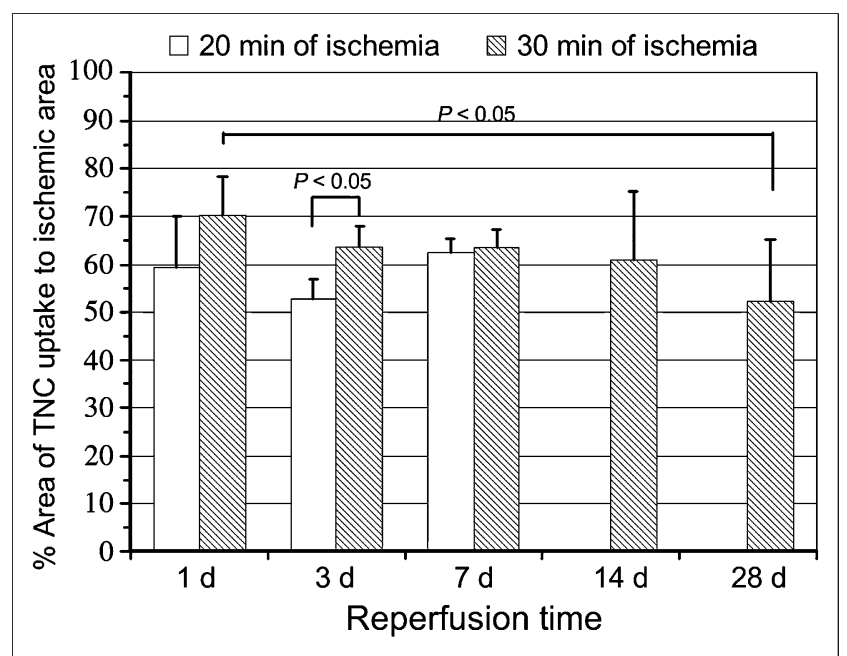

FIGURE 1. Time course of ${ }^{125}$ I-TNC-Ab uptake area against area at risk (\% area). In rats with 20 min of ischemia, area of tenascin-C uptake was similar among 1,3 , and $7 \mathrm{~d}$ after reperfusion. In rats with 30 min of ischemia, area was also similar among 1, 3, 7, 14 and $28 \mathrm{~d}$ except for significant difference only when day 1 was compared with day 28 .

\section{${ }^{125}$ I-TNC-Ab Uptake}

In visual analysis, significant ${ }^{125} \mathrm{I}-\mathrm{TNC}-\mathrm{Ab}$ uptake was observed predominantly in the midmyocardial to endomyocardial layers in the area at risk in rats with $20 \mathrm{~min}$ of ischemia at $1 \mathrm{~d}$ after reperfusion, and the intensity increased at $3 \mathrm{~d}$ followed by faint uptake at $7 \mathrm{~d}$. In rats with $30 \mathrm{~min}$ of ischemia, strong ${ }^{125}$ I-TNC-Ab uptake was also observed in the midmyocardial to endomyocardial layers in the area at risk at 1 and $3 \mathrm{~d}$ after reperfusion, followed by moderate uptake at 7 and $14 \mathrm{~d}$ and weak uptake at $28 \mathrm{~d}$ (Fig. 2).

In the quantitative analysis, the ${ }^{125} \mathrm{I}-\mathrm{TNC}-\mathrm{Ab}$ uptake ratio of the rats with $20 \mathrm{~min}$ of ischemia was $1.81 \pm 0.53$ at $1 \mathrm{~d}$ after reperfusion and increased at $3 \mathrm{~d}(2.46 \pm 0.79)$, followed by a significant reduction at $7 \mathrm{~d}(1.23 \pm 0.17, P<0.05$ vs. $3 \mathrm{~d})$. The uptake ratio of the rats with $30 \mathrm{~min}$ of ischemia at 1 and $3 \mathrm{~d}$ after reperfusion was equally high $(2.99 \pm 0.90$ and $2.71 \pm$ 0.80 , respectively); the uptake decreased at 7 and $14 \mathrm{~d}(1.94 \pm$ 0.23 and $2.06 \pm 0.37$, respectively) and then was weak at $28 \mathrm{~d}$ ( $1.47 \pm 0.27, P<0.005$ vs. $1 \mathrm{~d}, P<0.05$ vs. $3 \mathrm{~d})$. When rats with 20 and $30 \mathrm{~min}$ of ischemia were compared, ${ }^{125}$ I-TNCAb uptake in rats with 30 min of ischemia was higher at $1 \mathrm{~d}$ $(P<0.05)$ and $7 \mathrm{~d}(P<0.001)$ after reperfusion but was similar at $3 \mathrm{~d}$ (Fig. 3 ).

${ }^{125}$ I-labeled nonspecific antibody accumulated only slightly in the ischemic area (uptake ratio, $1.36 \pm 0.27$ ) in rats with $30 \mathrm{~min}$ of ischemia at $3 \mathrm{~d}$ after reperfusion. This uptake was significantly lower $(P<0.005)$ than that of ${ }^{125} \mathrm{I}-\mathrm{TNC}-\mathrm{Ab}(2.71 \pm 0.80)$.

\section{Biodistribution of ${ }^{125} \mid-T N C-A b$}

As shown in Table 1, uptake of ${ }^{125} \mathrm{I}-\mathrm{TNC}-\mathrm{Ab}$ in the infarcted area was $0.53 \pm 0.089,0.62 \pm 0.13$, and $0.27 \pm$ 


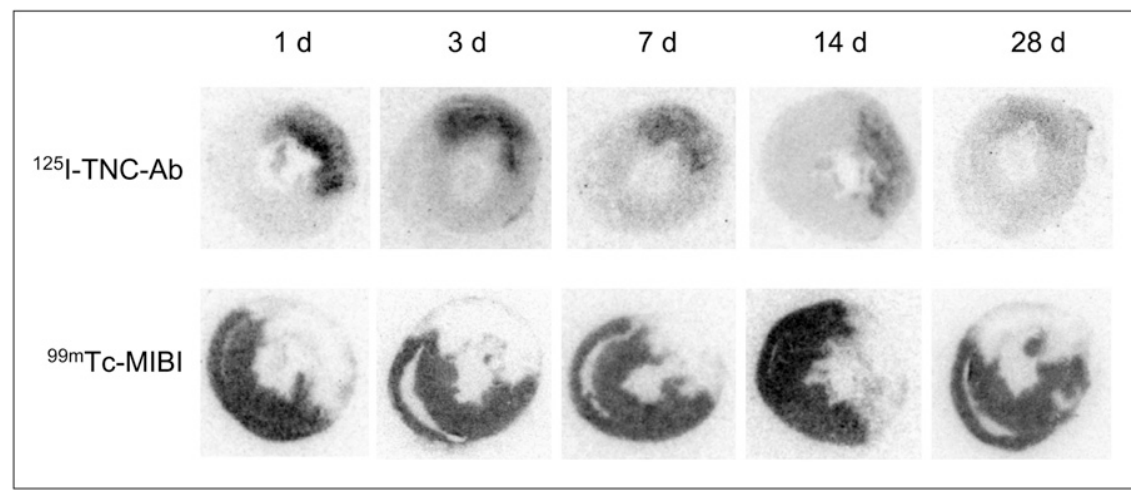

FIGURE 2. Autoradiography of 125|TNC-Ab and $99 \mathrm{mTC}-\mathrm{MIBI}$ in rats with 30 min of ischemia. Intense 125 I-TNC-Ab uptake was observed predominantly in midmyocardial to endomyocardial layers at 1 and $3 \mathrm{~d}$ after reperfusion. Reduced but significant ${ }^{125} \mathrm{I}-\mathrm{TNC}-\mathrm{Ab}$ uptake persisted at 7 and $14 \mathrm{~d}$, followed by obvious reduction of uptake at $28 \mathrm{~d}$.
0.11 percentage injected dose per gram of tissue at 1,5 , and $24 \mathrm{~h}$ after tracer injection, respectively. Because of the rapid blood clearance of ${ }^{125}$ I-TNC-Ab and good retention in infarcted areas, a decent infarcted area-to-blood ratio was obtained at 5 and $24 \mathrm{~h}$ after injection $(1.85 \pm 0.14$ and $4.8 \pm 1.0$, respectively), and the infarcted area-to-lung ratio at 5 and $24 \mathrm{~h}$ after tracer injection was $1.97 \pm 0.19$ and $4.9 \pm 1.2$, respectively, suggesting feasibility for in vivo imaging. However, liver uptake that was relatively high but less than double the infarcted area was observed until $24 \mathrm{~h}$.

\section{Histopathologic Findings}

The accumulation of ${ }^{125}$ I-TNC-Ab seen on autoradiography corresponded closely to the area with positive

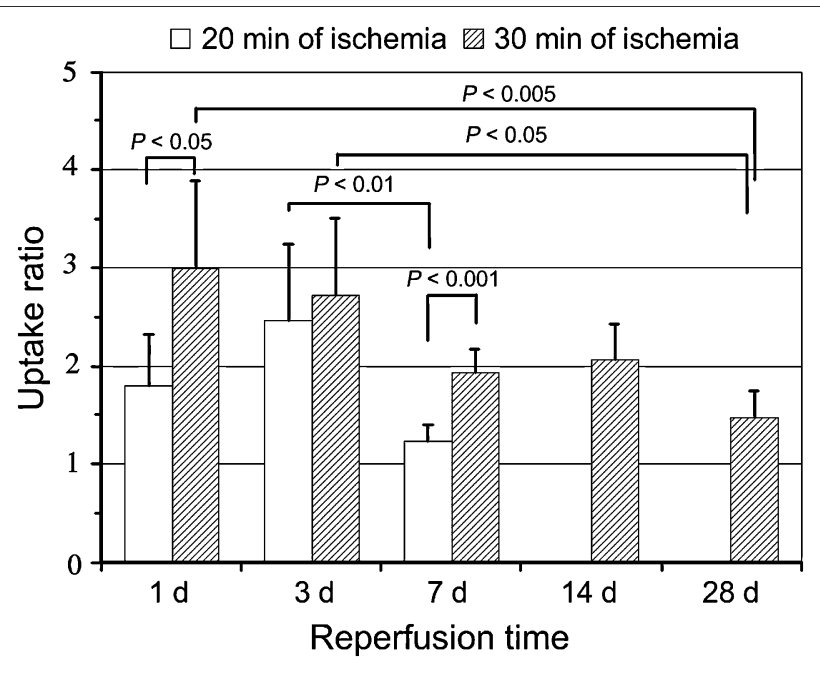

FIGURE 3. Time course of ${ }^{125}$ I-TNC-Ab uptake ratio in rats with 20 and $30 \mathrm{~min}$ of ischemia. ${ }^{125}$ I-TNC-Ab uptake ratio was calculated by dividing ${ }^{125}$ I-TNC-Ab count density in area at risk by that of nonischemic area. In rats with 20 min of ischemia, ${ }^{125}$ I-TNC-Ab uptake was significant at $1 \mathrm{~d}$ after reperfusion and peaked at $3 \mathrm{~d}$, followed by marked reduction at $7 \mathrm{~d}$. In rats with $30 \mathrm{~min}$ of ischemia, ${ }^{125}$ I-TNC$\mathrm{Ab}$ uptake ratio was highest at 1 and $3 \mathrm{~d}$, followed by reduction at 7 and $14 \mathrm{~d}$. Further reduction of uptake ratio was observed at $28 \mathrm{~d}$. immunohistopathologic staining by ${ }^{125} \mathrm{I}-\mathrm{TNC}-\mathrm{Ab}$. Representative cases are shown in Figure 4A.

One to $3 \mathrm{~d}$ after $30 \mathrm{~min}$ of ischemia and reperfusion, coagulation necrosis of cardiomyocytes and severe inflammatory cell infiltration were apparent in the infarcted area. Tenascin-C deposition and inflammation were observed throughout the infarcted area. Especially strong staining was noted at the border zone. No tenascin-C expression was detected in the intact area (Fig. 4B). Necrotic tissue was gradually replaced by granulation tissue, with attenuation of inflammation. Tenascin-C deposition gradually decreased and had almost disappeared on day 28 , when collagen fiberrich scar tissue had formed in the injured lesion.

\section{DISCUSSION}

The present study demonstrated the feasibility of imaging targeted at tenascin-C expression and documented for the first time, to our knowledge, spatiotemporal profiling of tenascin-C expression after myocardial ischemia and reperfusion. In myocardium with $20 \mathrm{~min}$ of ischemia and reperfusion, significant tenascin-C expression was observed inhomogeneously but predominantly in the midmyocardial to endomyocardial layers within the area at risk at $1 \mathrm{~d}$ after reperfusion, and the expression peaked at $3 \mathrm{~d}$, followed by a marked reduction of tenascin-C expression at $7 \mathrm{~d}$. However, when the ischemia was prolonged to $30 \mathrm{~min}$, the time course of the tenascin- $\mathrm{C}$ expression differed considerably; it peaked at 1 and $3 \mathrm{~d}$ after reperfusion, and a reduced but still significant expression persisted over 7-14 d, followed by an obvious decline in tenascin-C expression at $28 \mathrm{~d}$. We surmise that in the model of ischemia and reperfusion, the duration of coronary artery occlusion or the severity of ischemia is the most potent factor determining the degree and time course of tenascin- $\mathrm{C}$ expression. Histopathologic examination also demonstrated similar results obtained from autoradiography. More prolonged tenascin- $\mathrm{C}$ immunostaining was observed in the model of $30 \mathrm{~min}$ of ischemia and reperfusion than in the model of $20 \mathrm{~min}$ of ischemia.

A previous immunohistopathologic study on rats with permanent coronary ligation demonstrated interstitial positive tenascin- $\mathrm{C}$ immunoreactivity in the border zone 
TABLE 1. Biodistribution of ${ }^{125}$-TNC-Ab

\begin{tabular}{|c|c|c|c|}
\hline \multirow[b]{2}{*}{ Tissue } & \multicolumn{3}{|c|}{ Time after intravenous administration } \\
\hline & $1 \mathrm{~h}$ & $5 \mathrm{~h}$ & $24 \mathrm{~h}$ \\
\hline Blood & $0.59 \pm 0.069$ & $0.33 \pm 0.063$ & $0.057 \pm 0.020$ \\
\hline Heart (norm) & $0.22 \pm 0.018$ & $0.16 \pm 0.026$ & $0.059 \pm 0.035$ \\
\hline Heart (inf) & $0.53 \pm 0.089$ & $0.62 \pm 0.13$ & $0.27 \pm 0.11$ \\
\hline Liver & $0.97 \pm 0.43$ & $0.99 \pm 0.28$ & $0.34 \pm 0.099$ \\
\hline Lung & $0.48 \pm 0.39$ & $0.32 \pm 0.068$ & $0.054 \pm 0.012$ \\
\hline Spleen & $0.67 \pm 0.29$ & $0.41 \pm 0.039$ & $0.089 \pm 0.017$ \\
\hline Muscle & $0.063 \pm 0.052$ & $0.077 \pm 0.023$ & $0.020 \pm 0.0064$ \\
\hline Kidney & $1.23 \pm 0.71$ & $0.87 \pm 0.084$ & $0.24 \pm 0.11$ \\
\hline Heart (inf)/blood & $0.91 \pm 0.087$ & $1.85 \pm 0.14$ & $4.8 \pm 1.0$ \\
\hline Heart (inf)/lung & $1.20 \pm 0.71$ & $1.97 \pm 0.19$ & $4.9 \pm 1.2$ \\
\hline Heart (inf)/liver & $0.69 \pm 0.45$ & $0.66 \pm 0.16$ & $0.82 \pm 0.25$ \\
\hline
\end{tabular}

between infarcted and intact areas at $1 \mathrm{~d}$ after ligation. Approximately $3 \mathrm{~d}$ after ligation, necrotic myocardium began to be replaced by granulation tissue, and tenascin-C immunoreactivity was observed at the marginal zone and at developing fronts of granulation tissue around necrotic cardiomyocytes (5). However, in the present study with ischemia and reperfusion, ${ }^{125} \mathrm{I}-\mathrm{TNC}-\mathrm{Ab}$ accumulation and tenascin-C immunostaining were scattered throughout but restricted within the ischemic area at $1 \mathrm{~d}$ after reperfusion. Three days after reperfusion granulation, tissues were formed intricately in the midmyocardial to endomyocardial layers, with a vague border, and tenascin-C immunoreactivity was observed rather widely around granulation tissue. This finding can be explained by the presence of scattered surviving foci of myocardial tissue in the infarcted area because of tissue salvage by reperfusion.
After myocardial infarction, tenascin-C is markedly but transiently upregulated during the early phase of tissue repair, is predominantly produced by interstitial fibroblasts, and is localized in the border zone between infarcted and viable myocardium $(4,5)$. Tenascin- $\mathrm{C}$ may have a dual role in cardiac tissue repair and healing. Tenascin-C may loosen cardiomyocytes from the matrix, causing slippage of cardiomyocytes, and may facilitate inflammatory cell infiltration. Tenascin-C upregulates the transcription and activity of matrix metalloproteinases $(20,21)$, thereby promoting degradation of the extracellular matrix and slippage of myocytes within the ventricular wall, resulting in an increased risk of ventricular thinning and dilatation. On the other hand, tenascin-C may increase matrix production, resulting in reinforcement of the cardiac matrix $(5,6,22)$. A timely and proper degree of tenascin-C expression might
A 99mTC-MIBI 125-TNC-Ab Immunostaining
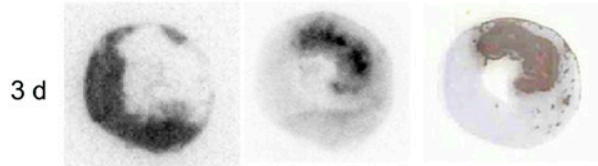

$7 d$

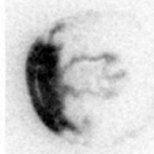

B

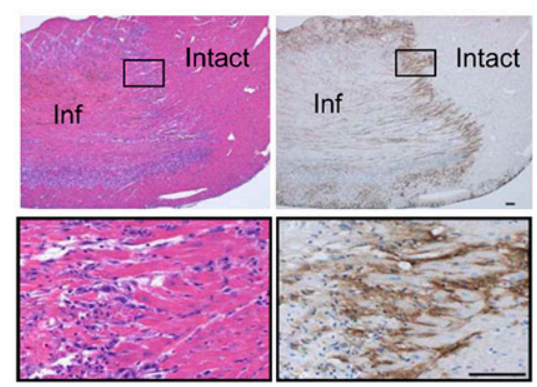

$3 d$

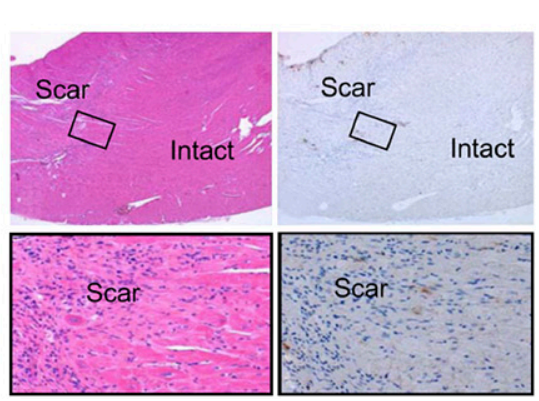

FIGURE 4. (A) Comparison of autoradiography of 125 -TNC-Ab and immunostaining. Distribution of 125 I-TNC-Ab and immunoreactivity were well colocalized in both models at 3 and $7 \mathrm{~d}$ after $30 \mathrm{~min}$ of ischemia and reperfusion. (B) Tenascin-C expression in myocardium at 3 and $28 \mathrm{~d}$ after $30 \mathrm{~min}$ of ischemia and reperfusion (hematoxylin-eosin staining on left and immunostaining for tenascin-C on right). Images at bottom are enlargements of boxed areas in images at top. Three days after reperfusion, coagulation necrosis of cardiomyocytes and severe inflammatory cell infiltration were seen in infarcted area (left images), and tenascin-C deposition was seen in infarcted area but not in intact area (right images), with especially strong staining noted at border zone. Twenty-eight days after reperfusion, collagen-rich scar tissue had formed in injured area (left images), and tenascin-C staining had largely disappeared (right images). Bar $=100 \mu \mathrm{m}$; inf $=$ infarcted area; intact $=$ intact area; scar $=$ scar tissue. 
benefit myocardial tissue repair, whereas prolonged overexpression of tenascin-C might interfere with sound tissue repair and cause inappropriate reconstruction of the infarcted ventricle, resulting in so-called ventricular remodeling. In patients with acute myocardial infarction, the peak serum tenascin-C level at $5 \mathrm{~d}$ after infarction is an important independent predictor of prognosis. A higher tenascin-C level is associated with a greater risk of left ventricular remodeling at 6 mo after infarction, indicating that overexpression of tenascin- $\mathrm{C}$ may aggravate ventricular remodeling (16). Furthermore, Frangogiannis et al. revealed that, in patients with severe chronic ischemic heart disease, dysfunctional myocardial segments expressing more tenascin-C showed significantly better functional recovery after bypass surgery than did segments without tenascin- $\mathrm{C}$ expression. Expression of tenascin- $\mathrm{C}$ in the dysfunctional segment may suggest that active continuous fibrosis is in progress in that area and may be beneficial for surgical interventional revascularization therapy to prevent ventricular remodeling (7).

The application of targeted radionuclide imaging of regional tenascin-C expression as proposed in the present study holds the potential to quantify the extent, amount, and localization of tenascin-C expression in vivo and to relate pathophysiologic events to tissue repair and remodeling after severe myocardial ischemia. Furthermore, the proposed imaging approach would provide the opportunity to monitor novel therapeutic interventions directed toward the reduction of extracellular matrix remodeling after myocardial infarction. Although the current study demonstrated changes in spatiotemporal ${ }^{125} \mathrm{I}-\mathrm{TNC}-\mathrm{Ab}$ accumulation after myocardial ischemia and reperfusion, further study should investigate the relationship between the degree or temporal prolongation of ${ }^{125} \mathrm{I}$-TNC-Ab uptake and ventricular dilatation or remodeling after myocardial infarction. In addition, assessments of whether interventional therapy to protect against myocardial damage and ventricular remodeling is related to changes in tenascin-C expression, and whether these really reflect the therapeutic effect, are also required.

In the present study, the anti-tenascin-C-antibody fragment was labeled so that the radioactivity would reveal inherent in vivo behaviors of the antibody fragment. The concordant spatial distribution of ${ }^{125}$ I-TNC-Ab and immunohistochemical staining confirmed that the ${ }^{125} \mathrm{I}-\mathrm{TNC}-\mathrm{Ab}$ had bound to tenascin-C expressed in rat myocardium. Specific accumulation of ${ }^{125} \mathrm{I}-\mathrm{TNC}-\mathrm{Ab}$ in the myocardial area with ischemia and reperfusion was confirmed by a significantly lower accumulation of the nonspecific antibody in the area at risk.

For future clinical application as a SPECT agent, ${ }^{123} \mathrm{I}-\mathrm{TNC}-\mathrm{Ab}$ or ${ }^{111}$ In-TNC-Ab is required. Labeling with ${ }^{123}$ I can easily be performed in the same way as for ${ }^{125} \mathrm{I}$, and ${ }^{111}$ In labeling has already been achieved $(9,17)$. Rapid blood clearance and retention of ${ }^{125}$ I-TNC-Ab in the infarcted area, resulting in a decent target-to-blood ratio and good target-to-lung ratio, indicate the feasibility of in vivo imaging at $5 \mathrm{~h}$ or later after tracer injection. However, relatively high liver uptake resulted in a low target-to-liver ratio $(0.66$ at $5 \mathrm{~h}$ and 0.82 at $24 \mathrm{~h})$, suggesting that careful interpretation might be required for myocardial uptake adjacent to the liver.

\section{CONCLUSION}

The present study demonstrated the feasibility of targeted ${ }^{125} \mathrm{I}-\mathrm{TNC}-\mathrm{Ab}$ imaging for serial assessment of regional expression of tenascin-C after myocardial ischemia and reperfusion. The study also demonstrated the spatiotemporal expression pattern after 20 and $30 \mathrm{~min}$ of ischemia and reperfusion. The data from this study implied that, by allowing visualization of tenascin-C expression, radiolabeled TNC Ab imaging may be a noninvasive way to monitor myocardial injury and its repair process after ischemia and reperfusion.

\section{ACKNOWLEDGMENTS}

This study was supported in part by 2 grants-in-aid for scientific research (C-20591437 and 19590813) from the Ministry of Education, Culture, Sports, Science, and Technology, Japan; by a research grant for intractable diseases from the Ministry of Health, Labor and Welfare of Japan; and by a grant from the Japan Foundation for the Promotion of the International Medical Center of Japan.

\section{REFERENCES}

1. White HD, Norris RM, Brown MA, Brandt PW, Whitlock RM, Wild CJ. Left ventricular end-systolic volume as the major determinant of survival after recovery from myocardial infarction. Circulation. 1987;76:44-51.

2. Chung CY, Murphy-Ullrich JE, Erickson HP. Mitogenesis, cell migration, and loss of focal adhesions induced by tenascin-C interacting with its cell surface receptor, annexin II. Mol Biol Cell. 1996;7:883-892.

3. Jones FS, Jones PL. The tenascin family of ECM glycoproteins: structure, function, and regulation during embryonic development and tissue remodeling. Dev Dyn. 2000;218:235-259.

4. Willems IE, Arends JW, Daemen MJ. Tenascin and fibronectin expression in healing human myocardial scars. J Pathol. 1996;179:321-325.

5. Imanaka-Yoshida $\mathrm{K}$, Hiroe $\mathrm{M}$, Nishikawa $\mathrm{T}$, et al. Tenascin-C modulates adhesion of cardiomyocytes to extracellular matrix during tissue remodeling after myocardial infarction. Lab Invest. 2001;81:1015-1024.

6. Imanaka-Yoshida K, Hiroe M, Yasutomi Y, et al. Tenascin-C is a useful marker for disease activity in myocarditis. J Pathol. 2002;197:388-394.

7. Frangogiannis NG, Shimoni S, Chang SM, et al. Active interstitial remodeling: an important process in the hibernating human myocardium. J Am Coll Cardiol. 2002;39:1468-1474.

8. Morimoto S, Imanaka-Yoshida K, Hiramitsu S, et al. Diagnostic utility of tenascin-C for evaluation of the activity of human acute myocarditis. $J$ Pathol. 2005;205:460-467.

9. Sato M, Toyozaki T, Odaka K, et al. Detection of experimental autoimmune myocarditis in rats by ${ }^{111} \mathrm{In}$ monoclonal antibody specific for tenascin-C. Circulation. 2002;106:1397-1402.

10. Tamura A, Kusachi S, Nogami K, et al. Tenascin expression in endomyocardial biopsy specimens in patients with dilated cardiomyopathy: distribution along margin of fibrotic lesions. Heart. 1996;75:291-294.

11. Tsukada B, Terasaki F, Shimomura H, et al. High prevalence of chronic myocarditis in dilated cardiomyopathy referred for left ventriculoplasty: expression of tenascin $\mathrm{C}$ as a possible marker for inflammation. Hum Pathol. 2009;40:1015-1022. 
12. Hessel MH, Bleeker GB, Bax JJ, et al. Reverse ventricular remodelling after cardiac resynchronization therapy is associated with a reduction in serum tenascin-C and plasma matrix metalloproteinase-9 levels. Eur J Heart Fail. 2007;9:1058-1063.

13. Milting H, Ellinghaus P, Seewald M, et al. Plasma biomarkers of myocardial fibrosis and remodeling in terminal heart failure patients supported by mechanical circulatory support devices. J Heart Lung Transplant. 2008;27: 589-596.

14. Terasaki F, Okamoto H, Onishi K, et al; Study Group for Intractable Diseases by a Grant from the Ministry of Health, Labor and Welfare of Japan. Higher serum tenascin-C levels reflect the severity of heart failure, left ventricular dysfunction and remodeling in patients with dilated cardiomyopathy. Circ J. 2007;71:327-330.

15. Fujimoto N, Onishi K, Sato A, et al. Incremental prognostic values of serum tenascin-C levels with blood B-type natriuretic peptide testing at discharge in patients with dilated cardiomyopathy and decompensated heart failure. $J$ Card Fail. 2009;15:898-905.

16. Sato A, Aonuma K, Imanaka-Yoshida K, et al. Serum tenascin-C might be a novel predictor of left ventricular remodeling and prognosis after acute myocardial infarction. J Am Coll Cardiol. 2006;47:2319-2325.
17. Odaka K, Uehara T, Arano Y, et al. Noninvasive detection of cardiac repair after acute myocardial infarction in rats by $111 \mathrm{In}$ Fab fragment of monoclonal antibody specific for tenascin-C. Int Heart J. 2008;49:481-492.

18. Taki J, Higuchi T, Kawashima A, et al. Effect of postconditioning on myocardial ${ }^{99 \mathrm{~m}} \mathrm{Tc}$-annexin-V uptake: comparison with ischemic preconditioning and caspase inhibitor treatment. J Nucl Med. 2007;48:1301-1307.

19. Wilbur DS, Hadley SW, Grant LM, Hylarides MD. Radioiodinated iodobenzoyl conjugates of a monoclonal antibody Fab fragment: in vivo comparisons with chloramine-T-labeled Fab. Bioconjug Chem. 1991;2:111-116.

20. Kalembeyi I, Inada H, Nishiura R, Imanaka-Yoshida K, Sakakura T, Yoshida T. Tenascin-C upregulates matrix metalloproteinase- 9 in breast cancer cells: direct and synergistic effects with transforming growth factor beta1. Int J Cancer. 2003; 105:53-60.

21. Nishiura R, Noda N, Minoura H, et al. Expression of matrix metalloproteinase-3 in mouse endometrial stromal cells during early pregnancy: regulation by interleukin-1alpha and tenascin-C. Gynecol Endocrinol. 2005;21:111-118.

22. Tamaoki M, Imanaka-Yoshida $\mathrm{K}$, Yokoyama $\mathrm{K}$, et al. Tenascin-C regulates recruitment of myofibroblasts during tissue repair after myocardial injury. Am J Pathol. 2005;167:71-80. 